\title{
A prognostic five immune-related long noncoding RNA signature for patients with colon cancer
}

\section{Kankan Zhao}

Zhujiang Hospital

Mengchuan Wang

Zhujiang Hospital

Houlong Kang

Zhujiang Hospital

Aiguo Wu ( $\nabla$ wagtyz@sina.com )

Zhujiang Hospital https://orcid.org/0000-0002-6793-8779

Primary research

Keywords: long non-coding RNA, colon cancer, prognostic biomarker, bioinformatics analysis

Posted Date: July 29th, 2020

DOl: https://doi.org/10.21203/rs.3.rs-47874/v1

License: (9) This work is licensed under a Creative Commons Attribution 4.0 International License. Read Full License 


\section{Abstract}

Background: Our study aimed to identify immune related long non-coding RNAs (LncRNAs) to serve as potential prognostic indicators and immune therapeutic targets in patients with colon cancer.

Methods: The Cancer Genome Atlas (TCGA) and Molecular Signatures Databases (MSigDB) database were used to identify immune related IncRNAs in patients with colon cancer. The least absolute shrinkage and selection operator (LASSO) analysis, and multivariate Cox proportional hazards regression analysis were employed to screen prognostic IncRNAs and construct immune-related multi-IncRNA signature. We used time-dependent receiver operating characteristic curve to assess the performance of this signature in colon cancer by calculating the area under the curve (AUC). Univariate and multivariate Cox regression analysis were performed to verify the independence of the prognostic value of this signature in colon cancer.

Results: Five immune related IncRNAs (AC025575.2, AL161729.4, ELFN1-AS1, LINC00513, MIR210HG) were found to be significantly associated with overall survival (OS) of patients with colon cancer. Then, we developed a five immune-related IncRNA signature. According to this signature, patients were ranked into a high risk group $(n=208)$ and a low risk group $(n=209)$. Kaplan-Meier curve and log-rank method showed that patients in high risk group had worse OS than patients in low risk group $(P=5.5644 \mathrm{e}-05)$. AUC for predicting 3 year survival and 5 year survival was 0.776 and 0.762 respectively, which indicated good performance of this signature. Finally, this five immune-related IncRNA signature was demonstrated to be independently associated with prognosis of patients with colon cancer.

Conclusion: We developed a five immune-related IncRNA signature as a prognostic biomarker for patients with colon cancer.

\section{Introduction}

Colon cancer ranks fourth in terms of incidence among all cancers according to statistics from International Agency for Research on Cancer (IARC) [1]. Despite advancement in diagnosis and treatment methods, the prognosis is still unsatisfactory. Tumor microenvironment (TME), one of the hallmarks of cancer, is defined as the cellular and physical environment surrounding the primary cancer [2]. The imbalance of immune status in TME plays an important role in the development and progression of cancer. Due to molecular alterations in the tumor cells and immune cells which are responsible for tumor cell recognition and killing, TME may exhibits immunosuppressive characteristics. Tumor immunosuppression may assist the immune escape of cancer cells, resulting in tumor progression [3, 4]. Immunotherapy targeted tumor immunosuppression mainly focus on blocking immune checkpoint receptors, enhancing $T$ cell recognition and neutralizing inhibitory molecules. However, immunological nonresponse and resistance are major obstacles to tumor eradication. Thus, it is essential to find novel biomarkers as potential prognostic indicators and immune therapeutic targets. 
Long noncoding RNAs (LncRNAs), a class of noncoding RNAs longer than 200 nucletides, can bind to chromatin, DNA sequences, RNA and protein, which makes them versatile in regulating gene expression at transcriptional, post-transcriptional and epigenetic levels [5, 6]. Dysregulated IncRNAs have been reported to be implicated in a variety of cancer types including colon cancer [7]. Many studies reported that IncRNAs are key regulators in the tumor microenvironment, epithelial-mesenchymal transition (EMT), microbiota, metabolism, immune cell differentiation and immune system function in cancer [8-10]. Therefore, we aim to screen potential and valuable immune related IncRNAs to serve as potential prognostic indicators and immune therapeutic targets.

In the present study, we identified immune related IncRNAs in patients with colon cancer by applying The Cancer Genome Atlas (TCGA) and Molecular Signatures Databases (MSigDB). In addition, we developed a novel five immune-related IncRNA signature that could predict prognosis of patients with colon cancer. We also demonstrated that the five immune-related IncRNA signature was independently associated with overall survival (OS) of patients with colon cancer. Our analysis suggests potential roles of IncRNAs in colon cancer pathogenesis and progression.

\section{Materials And Methods}

The gene expression quantification data (HTSeq-FPKM) and corresponding clinic data of patients with colon cancer were retrieved from the TCGA colon adenocarcinoma (COAD) project (http://cancer genome.nih.gov). We excluded patients without IncRNA sequence data and patients having a survival time of 0 day. The immune related mRNAs were downloaded from the MSigDB (http://www.broadinstitute.org/gsea/msigdb/index.jsp, version 7.0) [11]. To identify immune related IncRNAs in colon cancer, we performed Person correlation analysis for each of the immune related mRNAs with IncRNAs based on the sequence data of the TCGA COAD project. The IncRNAs with IPerson coefficient| $>0.7$ were considered to be immune related IncRNAs.

\section{Identification of immune related IncRNA signature in colon cancer}

These immune related IncRNAs entered into the least absolute shrinkage and selection operator (LASSO) method analysis to select the best candidates by employing glmnet package in $\mathrm{R}$ software (version3.6.1) [12]. Then, we conducted multivariate Cox proportional hazards regression analysis to develop the immune-related multi-IncRNA signature. Based on this immune-related IncRNA signature, we calculated the risk score for each patient by integrating the IncRNAs expression profiles and relative multivariate Cox regression coefficient. Using the median risk score, patients were devided into a high risk group and low risk group. The Kaplan-Meier curve and log-rank method were performed to evaluate the OS difference between the high-risk group and low-risk group. Distribution of risk scores, survival status of patients with colon cancer, and expression profiles of prognostic IncRNAs were exhibited using R software. We used time-dependent receiver operating characteristic (ROC) curve to assess the sensitivity and specificity of the immune-related IncRNA signature by calculating the area under the curve (AUC) $[13,14]$. 
To verify the independence of the prognostic value of the immune-related IncRNA signature based risk score and clinicopathological factors (including age, sex, TNM stage, pathological stage), univariate and multivariate Cox regression analysis were performed to explore their associations with OS of patients with colon cancer. P-value $<0.05$ was considered to be statistically significant.

\section{Results}

\section{Identification of immune related IncRNAs in colon cancer}

A total of 417 patients with baseline data and gene expression quantification data were included. The detailed clinicopathological information was presented in Table 1. Based on the data of MSigDB (Immune system process M13664, Immune response M19817) and expression quantification data of TCGA COAD project, we identified 311 immune related IncRNAs in patients with colon cancer by applying Person correlation analysis (Table S1). 
Table 1

Clinicopathological characteristics of patients with colon cancer

\begin{tabular}{|c|c|}
\hline Characteristics & Number of cases (\%) \\
\hline Age & 118(28.3) \\
\hline$<60$ & 299(71.7) \\
\hline \multicolumn{2}{|l|}{$\geq 60$} \\
\hline \multicolumn{2}{|l|}{ Gender } \\
\hline Female & 193(46.3) \\
\hline Male & $224(53.7)$ \\
\hline \multicolumn{2}{|l|}{ T stage } \\
\hline Tis & $1(0.2)$ \\
\hline $\mathrm{T} 1$ & $9(2.2)$ \\
\hline $\mathrm{T} 2$ & $74(17.7)$ \\
\hline T3 & $284(68.1)$ \\
\hline $\mathrm{T} 4$ & $49(11.8)$ \\
\hline \multicolumn{2}{|l|}{$N$ stage } \\
\hline NO & $247(59.2)$ \\
\hline $\mathrm{N} 1$ & $98(23.5)$ \\
\hline N2 & $72(17.3)$ \\
\hline \multicolumn{2}{|l|}{ M stage } \\
\hline Mo & $311(74.6)$ \\
\hline M1 & $57(13.7)$ \\
\hline $\mathrm{Mx}$ & $49(11.8)$ \\
\hline \multicolumn{2}{|l|}{ Risk score } \\
\hline Low risk & $209(50.1)$ \\
\hline High risk & 208(49.9) \\
\hline
\end{tabular}

\section{Identification of immune related IncRNA signature in colon cancer}

These immune related IncRNAs were further assessed for their association with prognosis of patients with colon cancer. We identified 19 IncRNAs using LASSO Cox analysis (Figure 1). Then, multivariable Cox proportional hazards regression analysis were performed to identify the optimal immune related 
IncRNAs. The results showed that five IncRNAs (AC025575.2, AL161729.4, ELFN1-AS1, LINC00513, MIR210HG) significantly affected OS of patients with colon cancer (Figure 2). We constructed a immune related signature by integrating the five IncRNAs expression profiles and corresponding Cox regression coefficient. According to this five immune-related IncRNA signature, we calculated the risk score for each patients and ranked them into a high risk group $(n=208)$ and a low risk group $(n=209)$ based on the median of risk score (Table S2). The Kaplan-Meier curve showed patients in high risk group have significantly worse OS than patients in low risk group $(P=5.5644 \mathrm{e}-05$, Figure 3$)$. Distribution of risk score, survival status of patients with colon cancer, and the expression profiles of the five prognostic IncRNAs were shown in Figure 4. As Figure 4 showed, the mortality was much higher for patients with high risk score than those with low risk score and patients in the high risk group have a tendency to higher expression of AC025575.2, AL161729.4, ELFN1-AS1 and MIR210HG, and lower expression of LINC00513. The prognostic power of the five-IncRNA signature was evaluated by calculating the area under the ROC curve (AUC). The results showed that the AUC of five-IncRNA signature for predicting 3 year survival and 5 year survival was 0.776 and 0.762 respectively, which indicated good performance (Figure 5).

\section{Independence of the five-IncRNA signature with prognosis of patients with colon cancer}

The results of univariate Cox regression analysis indicated that the five-IncRNA signature based risk score, age, stage, $\mathrm{T}$ stage, $\mathrm{N}$ stage, $\mathrm{M}$ stage were significantly related to OS of patients with colon cancer (Figure 6A). Multivariate Cox regression analysis showed that age, $T$ stage, and five-IncRNA signature independently related to OS of patients with colon cancer (Figure 6B).

\section{Discussion}

In the present study, we identified 311 immune related IncRNAs in patients with colon cancer. Then, using Lasso analysis and Cox regression, we constructed a five immune-related IncRNA signature with good performance. According to the five immune-related IncRNA signature, patients with colon cancer can be classified into a high risk group and a low risk group with significantly different OS. Finally, this five immune-related IncRNA signature were demonstrated to independently predict the prognosis of patients with colon cancer through univariate and multivariate Cox regression analysis.

Among the five IncRNAs, AC025575.2, and AL161729.4 are novel transcript. The functions of them are unknown. ELFN1-AS1 is antisense IncRNA. It has been reported that ELFN1-AS1 was highly expressed in many cancer types including lung cancer, uterus cancer, and ovarian cancer [15]. In addition, Dong et al. found that ELFN1-AS1 promoted COAD cell proliferation and migration, and might be a potential target for COAD treatment [16]. Our results are consistent with their findings that ELFN1-AS1 played tumor promoting role in COAD. LINC00513 is intergenic IncRNA and played important roles in lupus pathogenesis by promoting IFN signaling pathway [17]. However, little is known about the role of LINC00513 in cancer. MIR210HG belongs to microRNA non-coding host gene. Many studies have reported the functions of MIR210HG in various cancer types. Wang et al. found that MIR210HG promoted cervical cancer cell proliferation, invasion and epithelial mesenchymal transition (EMT) through regulating MIR210HG/miR-503-5p/TRAF4 axis [18]. MIR210HG could sponge miR-126-3p to promote 
breast cancer cell invasion and metastasis through regulating mucin-1c and EMT pathway [19]. Similarly, MIR210HG could sponge miR-503 to function as oncogenic IncRNA in osteosarcoma [20]. MIR210HG was also demonstrated to facilitate progress of lung cancer [21], glioma [22], and hepatocellular carcinoma [23]. Moreover, two articles using bioinformatics methods identified MIR210HG was associated with poor OS of patients with colon cancer [24,25], which was in accordance with the results of our study. Our results imply potential functions of the five immune-related IncRNA signature in the development of colon cancer, suggesting them as important biomarkers and therapeutic targets. However, the predicted functions of the five-IncRNA signature could not only be interpreted through this method. Thus, studies remain need to be conducted to validate the biological function of these five IncRNAs and explore additional targets and strategies for treatment. Admittedly, our study has some limitations because it was based on the high-throughput RNA-sequencing profiles and data analysis only. The roles of these five IncRNA deserve further in vitro and in vivo studies. In conclusion, we developed the five immune-related IncRNA signature as a potential prognostic indicator for patients with colon cancer.

\section{Declarations}

\section{Ethical approval and informed consent}

Not applicable.

\section{Consent for publication}

Not applicable.

\section{Availability of data and material}

All data and material used to support the findings of this article are included within this article and supplementary information files.

\section{Competing interest}

The authors declare that they have no competing interests.

\section{Funding}

There was no funding for this article.

\section{Author's contributions}

$\mathrm{KZ}$ and MW performed the research, analyzed data, and drafted the manuscript. HK participated in data analysis. AW participated in research design. All authors read and approved the final manuscript.

\section{Acknowledgements}

Not applicable. 


\section{References}

1. Bray F, Ferlay J, Soerjomataram I, Siegel RL, Torre LA, Jemal A. "Global cancer statistics 2018 : GLOBOCAN estimates of incidence and mortality worldwide for 36 cancers in 185 countries". CA Cancer J Clin. 2018;68(6):394-424.

2. Hanahan D, Weinberg RA. "Hallmarks of cancer: the next generation," $C E L L$, vol. 144, no. 5, pp. 646674, 2011.

3. Yu WD, Wang H, He QF, Xu Y, Wang XC. "Long noncoding RNAs in cancer-immunity cycle". JOURNAL OF CELLULAR PHYSIOLOGY. 2018;233(9):6518-23.

4. Luo Y, Yang J, Yu J, et al. "Long Non-coding RNAs: Emerging Roles in the Immunosuppressive Tumor Microenvironment". Front Oncol. 2020;10:48.

5. Rinn JL, Chang HY. "Genome regulation by long noncoding RNAs". Annu Rev Biochem. 2012;81:14566.

6. Engreitz JM, Haines JE, Perez EM, et al. "Local regulation of gene expression by IncRNA promoters, transcription and splicing". NATURE. 2016;539(7629):452-5.

7. Gibb EA, Brown CJ, Lam WL. "The functional role of long non-coding RNA in human carcinomas". Molecular Cancer. 2011;10:38.

8. Chen D, Lu T, Tan J, Li H, Wang Q, Wei L. "Long Non-coding RNAs as Communicators and Mediators Between the Tumor Microenvironment and Cancer Cells". Front Oncol. 2019;9:739.

9. Del VF, Lee GH, Hawezi J, et al. "Long non-coding RNAs within the tumour microenvironment and their role in tumour-stroma cross-talk". CANCER LETTERS. 2018;421:94-102.

10. Denaro N, Merlano MC, Lo NC. "Long noncoding RNAs as regulators of cancer immunity". Mol Oncol. 2019;13(1):61-73.

11. Subramanian A, Tamayo P, Mootha VK, et al. "Gene set enrichment analysis: a knowledge-based approach for interpreting genome-wide expression profiles". Proc Natl Acad Sci U S A. 2005;102(43):15545-50.

12. Friedman J, Hastie T, Tibshirani R. "Regularization Paths for Generalized Linear Models via Coordinate Descent". J Stat Softw. 2010;33(1):1-22.

13. Blanche P, Dartigues JF, Jacqmin-Gadda H. "Estimating and comparing time-dependent areas under receiver operating characteristic curves for censored event times with competing risks". STATISTICS IN MEDICINE. 2013;32(30):5381-97.

14. Heagerty PJ, Lumley T, Pepe MS, "Time-dependent ROC curves for censored survival data and a diagnostic marker," BIOMETRICS, vol. 56, no. 2, pp. 337-344, 2000.

15. Polev DE, Karnaukhova IK, Krukovskaya LL, Kozlov AP, "ELFN1-AS1: a novel primate gene with possible microRNA function expressed predominantly in human tumors," Biomed Research International, vol. 2014, pp. 398097, 2014.

16. Dong L, Ding C, Zheng T, et al. "Extracellular vesicles from human umbilical cord mesenchymal stem cells treated with siRNA against ELFN1-AS1 suppress colon adenocarcinoma proliferation and 
migration". American Journal of Translational Research. 2019;11(11):6989-99.

17. Xue Z, Cui C, Liao Z, et al. "Identification of LncRNA Linc00513 Containing Lupus-Associated Genetic Variants as a Novel Regulator of Interferon Signaling Pathway". Front Immunol. 2018;9:2967.

18. Wang $\mathrm{AH}$, Jin $\mathrm{CH}$, Cui GY, et al, "MIR210HG promotes cell proliferation and invasion by regulating miR-503-5p/TRAF4 axis in cervical cancer" Aging (Albany NY), vol. 12, pp. 2020.

19. Li XY, Zhou LY, Luo H, et al. "The long noncoding RNA MIR210HG promotes tumor metastasis by acting as a ceRNA of miR-1226-3p to regulate mucin-1c expression in invasive breast cancer". Aging. 2019;11(15):5646-65.

20. Li J, Wu QM, Wang XQ, Zhang CQ. "Long Noncoding RNA miR210HG Sponges miR-503 to Facilitate Osteosarcoma Cell Invasion and Metastasis". DNA AND CELL BIOLOGY. 2017;36(12):1117-25.

21. Kang X, Kong F, Huang K, et al. "LncRNA MIR210HG promotes proliferation and invasion of nonsmall cell lung cancer by upregulating methylation of CACNA2D2 promoter via binding to DNMT1". Onco Targets Ther. 2019;12:3779-90.

22. Min W, Dai D, Wang J, et al. "Long Noncoding RNA miR210HG as a Potential Biomarker for the Diagnosis of Glioma". PLoS One. 2016;11(9):e160451.

23. Wang Y, Li W, Chen X, Li Y, Wen P, Xu F, "." "MIR210HG predicts poor prognosis and functions as an oncogenic IncRNA in hepatocellular carcinoma. BIOMEDICINE PHARMACOTHERAPY. 2019;111:1297-301.

24. Ruan Z, Xu Z, Li Z, Lv Y. "Integral analyses of survival-related long non-coding RNA MIR210HG and its prognostic role in colon cancer. Oncology Letters. 2019;18(2):1107-16.

25. He Z, Dang J, Song A, Cui X, Ma Z, Zhang Z. "Identification of LINC01234 and MIR210HG as novel prognostic signature for colorectal adenocarcinoma". JOURNAL OF CELLULAR PHYSIOLOGY. 2019;234(5):6769-77.

\section{Figures}

A



B

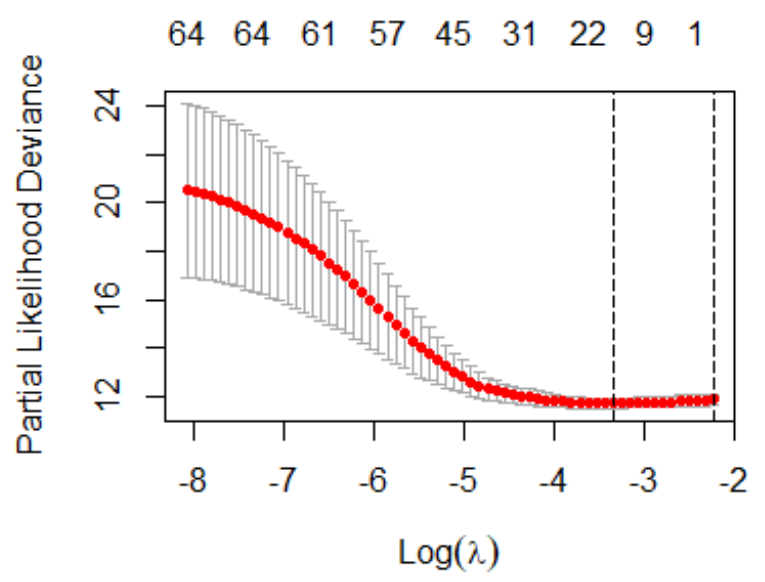


Figure 1

Selection of candidate IncRNAs through LASSO analysis. (A) LASSO coefficient profiles of differentially expressed IncRNAs. (B) "Leave-one-out-cross-validation" for parameter selection in the LASSO model. LASSO, least absolute shrinkage and selection operator.

\section{Hazard ratio}



\section{Figure 2}

Forest plot of the five immune related IncRNAs for affecting the prognosis of patients with colon cancer according to the results of multivariable Cox proportional hazards regression analysis. 


\section{Surival curve of risk score $(P=5.564 \mathrm{e}-05)$}

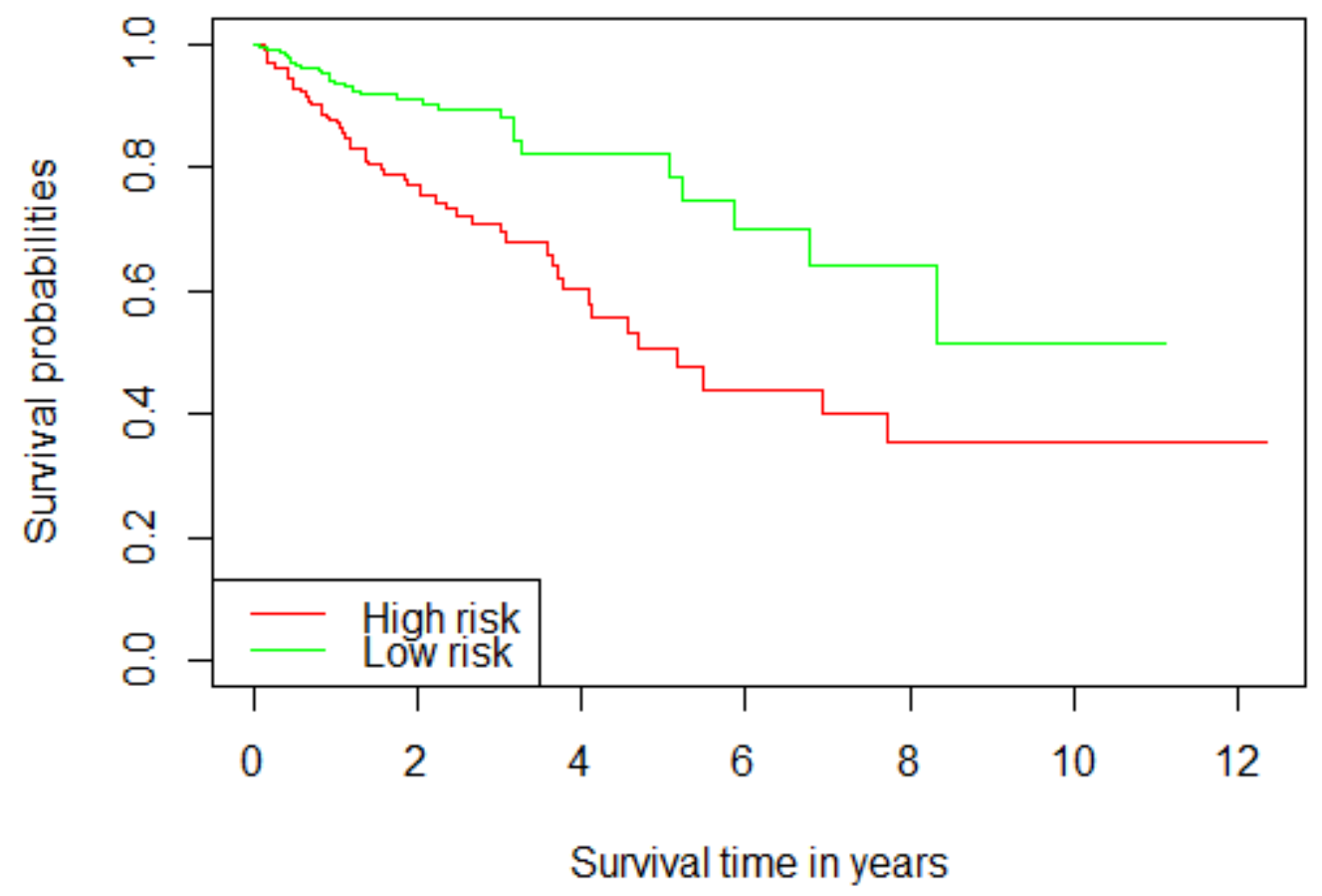

Figure 3

Kaplan-Meier plots of OS among patients in the high risk group and low risk group according to the five immune-related IncRNA signature. OS, overall survival. 

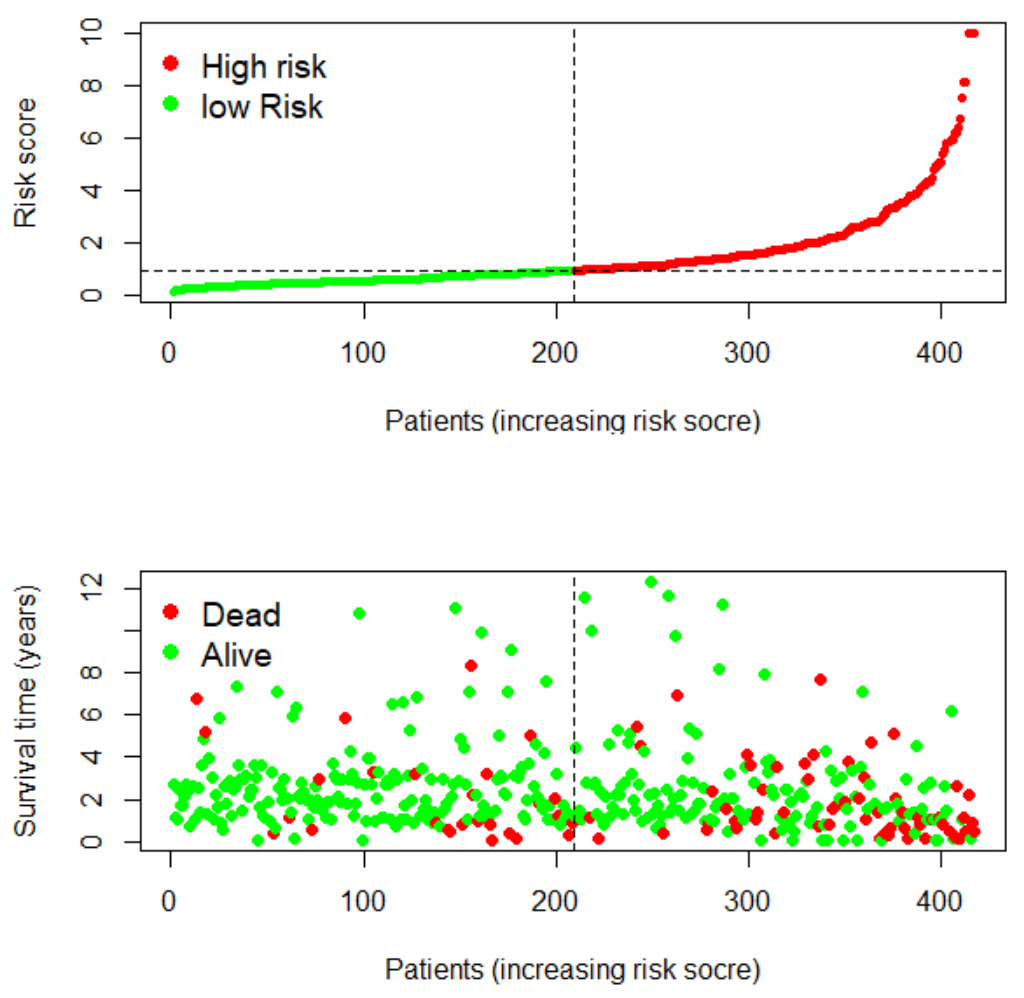

Low risk High risk

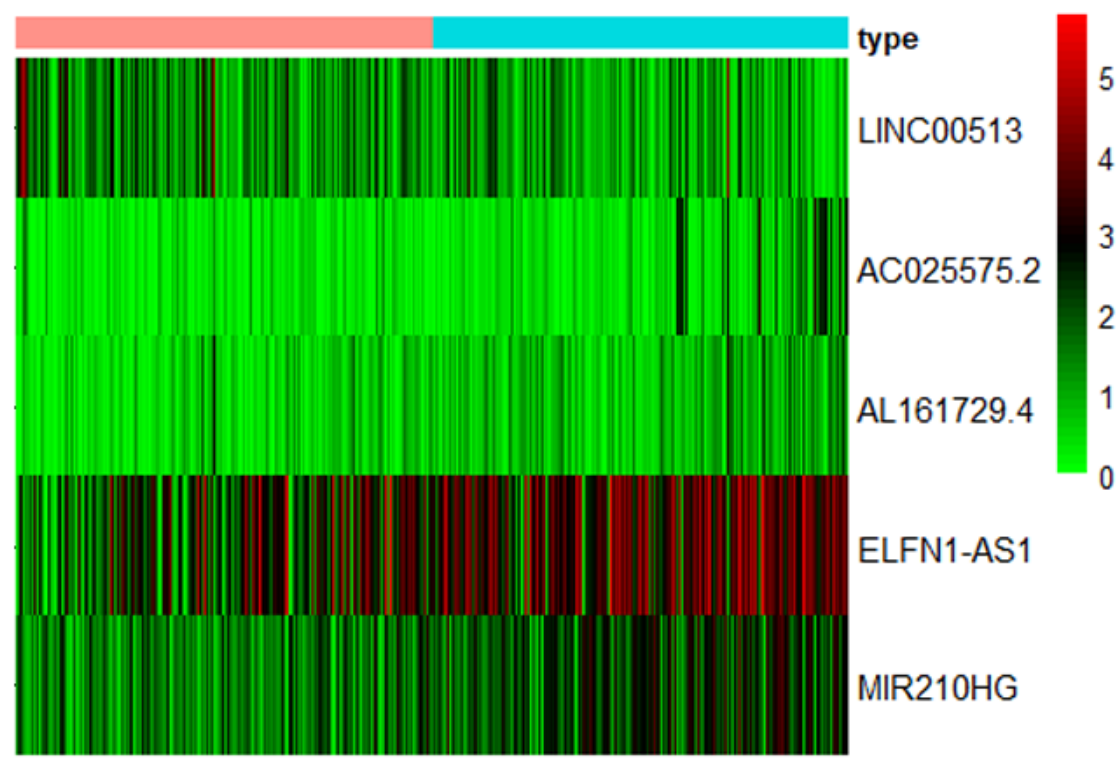

\section{Figure 4}

Risk score distribution, survival status and expression profiles of the five IncRNAs among patients in the high risk group and low risk group according to the five immune-related IncRNA signature. 


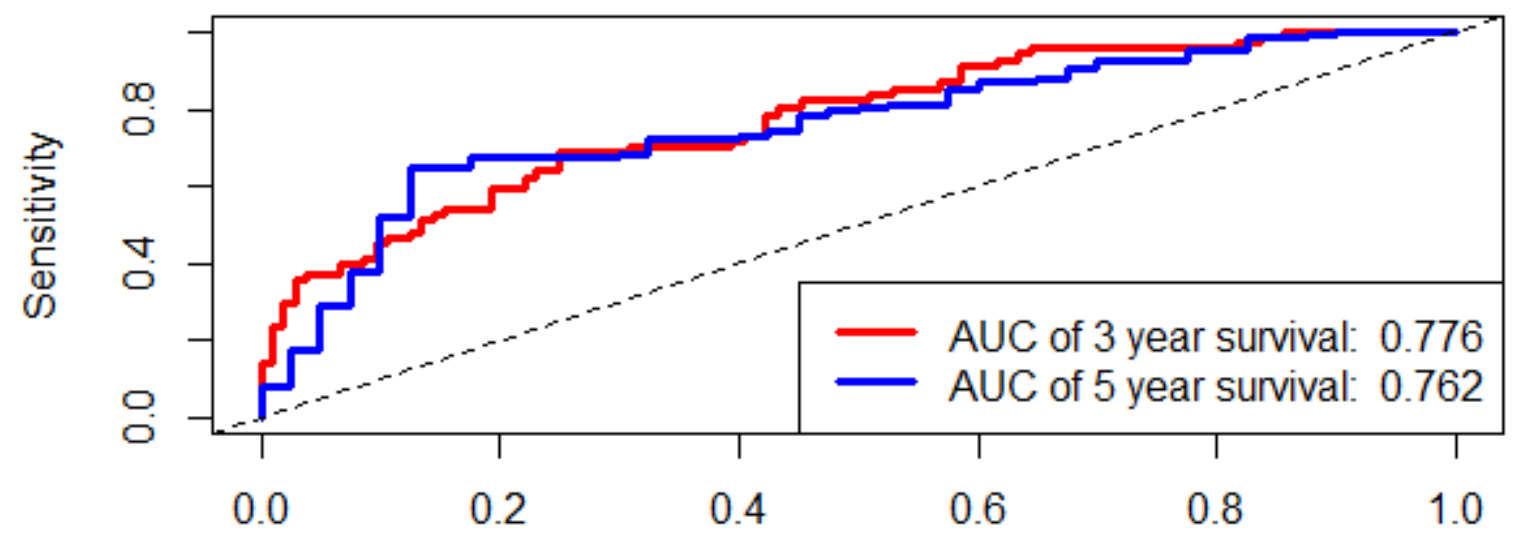

1-Specificity

Figure 5

ROC curve of the five immune related IncRNA signature for predicting 3 year survival and 5 year survival of patients with colon cancer. ROC, receiver operating characteristic.

A

Univariate Cox regression analysis

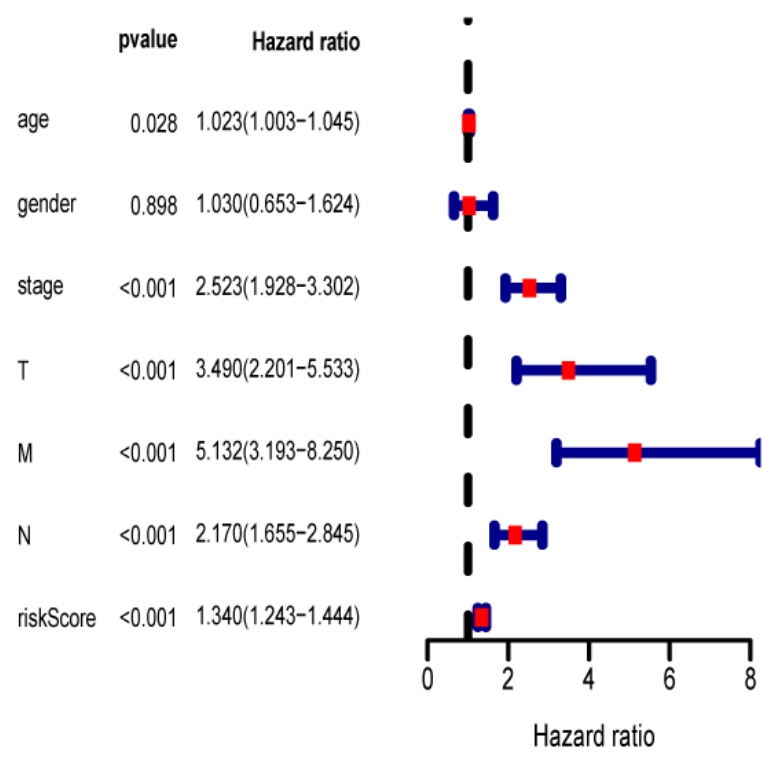

B

Multivariate Cox regression analysis

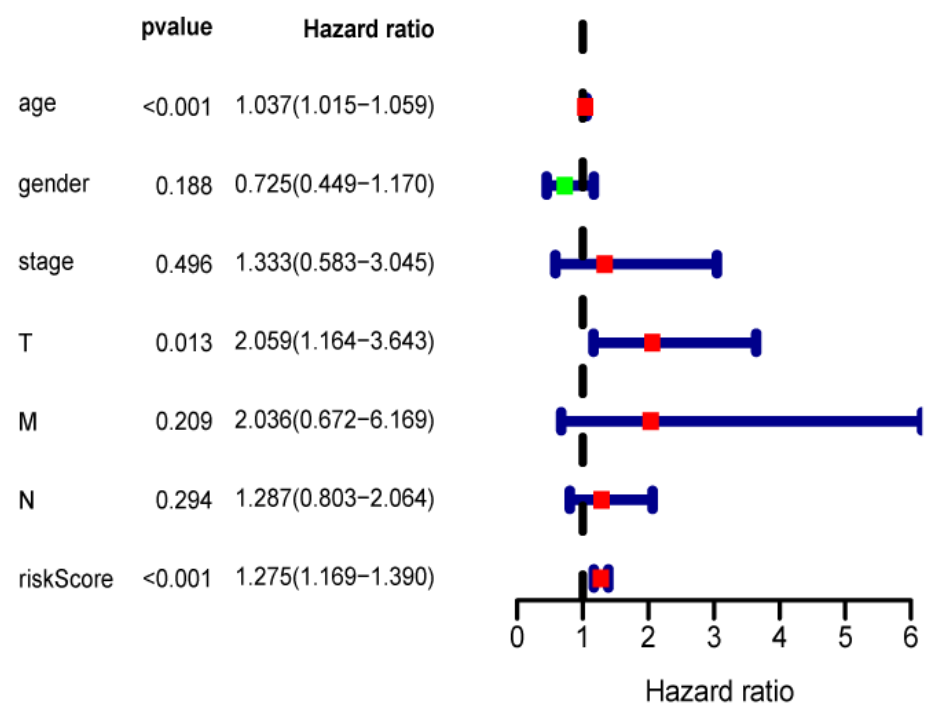

\section{Figure 6}

Forest plots of the five immune related IncRNA signature and clinicopathological factors for predicting prognosis of patients with colon cancer. (A) Univariate Cox proportional hazards regression analysis. (B) Multivariate Cox proportional hazards regression analysis. 


\section{Supplementary Files}

This is a list of supplementary files associated with this preprint. Click to download.

- SupplementaltableS1.docx

- SupplementaltableS2.docx 